\title{
High Risk of Surgical Glove Perforation From Surgical Rotatory Instruments
}

\author{
Ashton H. Goldman MD, Emanuel Haug BS, John R. Owen MS, \\ Jennifer S. Wayne PhD, Gregory J. Golladay MD
}

Received: 1 March 2016/Accepted: 14 June 2016/Published online: 23 June 2016

(C) The Association of Bone and Joint Surgeons \& 2016

\begin{abstract}
Background Surgical gloves can be damaged during the course of a procedure, which can place the surgeon and patient at risk. Glove perforation may not always be readily apparent, and determining the risk factors for glove perforation can aid the surgeon in deciding when a glove change is advisable. Time of wear and needle sticks have been well studied; however, other mechanisms including mechanical stress from surgical equipment have had limited evaluation to date.

Questions/purposes We evaluated the risk of glove perforation in gloves that were caught in a surgical rotatory device (such as drills and reamers). The aims of our study were (1) to determine the percentage of undetected
\end{abstract}

The institution of the authors has received, during the study period, funding from MEDARVA Healthcare (Richmond, VA, USA).

One author (GJG), or a member of his immediate family, has or may receive payments or benefits outside the submitted work from OrthoSensor Inc (Dania Beach, FL, USA), Stryker Inc. (Mahwah, NJ, USA), and Cayenne Medical (Scottsdale, AZ, USA).

Each author (AHG, EH, JRO, JSW, GJG) certifies that he or she has no commercial associations (eg, consultancies, stock ownership, equity interest, patent/licensing arrangements, etc) that might pose a conflict of interest in connection with the submitted article. Partial financial support was received from MEDARVA Healthcare, Richmond, VA.

All ICMJE Conflict of Interest Forms for authors and Clinical Orthopaedics and Related Research ${ }^{\mathbb{R}}$ editors and board members are on file with the publication and can be viewed on request.

A. H. Goldman, E. Haug, J. R. Owen, J. S. Wayne ( $₫)$, G. J. Golladay

Orthopaedic Research Laboratory, Departments of Orthopaedic Surgery \& Biomedical Engineering, Virginia Commonwealth University, P.O. Box 843067, Richmond, VA 23284-3067, USA

e-mail: jwayne@vcu.edu microperforations after entanglement on a rotatory tool during orthopaedic procedures, (2) to determine which kinds of rotatory devices most commonly cause such microperforations, and (3) to assess whether time of wear had an effect on the risk of perforation.

Methods From July 2014 to September 2015, 33 gloves were obtained from all orthopaedic subspecialties at our Level I trauma center if they were caught in a rotatory device greater than one revolution. Time of glove wear and location of the glove that was caught in a rotatory device were recorded. After an evaluation for macroperforations ( $\geq 5 \mathrm{~mm}$ ), the gloves were evaluated for microperforations $(<5 \mathrm{~mm})$ via the American Society for Testing and Materials (ASTM) one-liter load test. Time of wear was compared among gloves with macroperforations, microperforations, and no perforations.

Results The 33 gloves obtained came from 33 procedures. Seventeen of 33 (52\%) gloves had perforations. Seven of the 17 perforated gloves had macroperforations while 10 had microperforations. Eleven of 33 entanglements were caught by drills, nine by reamers, eight by Kwires, and the remaining five gloves were caught by various other instruments. Eight of 17 perforations were caused by drills, three by reamers, three by $\mathrm{K}$-wires, and three by various other instruments. The average time of wear was 58 minutes which did not differ with pattern of glove damage. Conclusion Surgical gloves caught in a rotatory power instrument are likely to have been perforated, regardless of the amount of time that they had been worn. Visual inspection appears to be an inadequate test of glove integrity. When a glove becomes entangled in a rotary instrument such as a drill, pin, or reamer, a surgeon should change the gloves regardless of whether he or she believes a perforation is present.

Level of Evidence Level IV, therapeutic study. 


\section{Introduction}

Surgical gloves provide the only physical barrier between the surgeon's hands and the patient to minimize contamination and disease transmission. It is well documented that perforations occur frequently during various procedures $[1,2,6]$. Perforations can increase the risk of surgical-site infection, and expose the surgeon to blood-borne diseases such as HIV, hepatitis $\mathrm{C}$ virus, and hepatitis $\mathrm{B}$ virus $[10,14]$. Understanding the clinical situations in which perforations occur can aid the surgeon in taking steps to minimize the risk of infection to the patient and to minimize the risk of viral exposure to the surgeon.

Some of the highest perforation percentages are seen in orthopaedic surgical procedures $[1,2]$. The use of power tools, handling sharp bone, and working in deep cavities all influence the risk of glove perforations in orthopaedic surgery [20]. Double gloving has been recommended in orthopaedic procedures to ameliorate the risk of perforation but compliance is not universal [7, 18]. Duration of wear, degree of soilage, and other risk factors for glove perforation also have been reported [2,16]. Identification of specific surgical steps or situations in which glove contamination is high may allow the surgeon to recognize when a glove change is indicated. McCue et al. [12] reported that $19 \%$ of gloves became contaminated during draping for THA, and they recommend that the surgical team don a third set of gloves for draping and then remove the outer pair before the start of surgery. Other recommendations have been made regarding time of wear or scheduled changes before certain steps such as implant placement $[4,16,18]$. Although damage to gloves from needle stick injury is well documented [15], little has been reported regarding other mechanisms of glove damage during surgical procedures.

Shear stress on gloves has been documented as a potential risk for glove perforation [5]. Arthroscopic knot tying is an example of shear-induced stress leading to a perforation risk of as much as 34\% [9]. Gloves can be subject to shear when they become entangled in a rotatory power device intraoperatively. To our knowledge, entanglement in rotatory tools has not been reported as a mechanism of glove damage. A common practice is to visually inspect a glove after entanglement occurs and to change the glove only if visually evident damage is present; however, how often this approach correctly identifies a perforation has not been evaluated, and this seemed possible to us.

Therefore, the aims of our study were (1) to determine the percentage of undetected microperforations after entanglement on a rotatory tool during orthopaedic procedures, (2) to determine which kinds of rotatory devices most commonly cause such microperforations, and (3) to assess whether time of wear had an effect on the risk of perforation.

\section{Methods}

This study does not involve human or animal data and requires no ethical board approval. From July 2014 until September 2015, orthopaedic surgeons and residents at our Level I trauma center were asked to submit gloves that were entangled intraoperatively and spun greater than one revolution in a rotatory power device. Study protocol and recruitment notices were placed in each operating room, and operating room personnel and surgeons were educated regarding study design and collection procedures. Surgeons from all orthopaedic subspecialties were encouraged to submit gloves. Our departmental practice is to routinely double glove for most orthopaedic procedures. The selection of glove type is according to surgeon preference. Potentially damaged outer gloves were submitted on a voluntary, anonymous basis at the time of entanglement. Data were collected to estimate the time of wear, the instrument used, which finger was involved, and if the patient had any known transmittable infectious disease. Gloves used on patients with known transmittable infectious disease or an active infection were excluded from the study. Thirty-three gloves were acquired during the collection period. The per-procedure risk could not be calculated, since we had no reliable means to determine the number of cases during the study period in which rotatory instruments were used. Likewise, anonymous submission precluded any analysis by subspecialty in orthopaedic surgery. Gloves were collected, refrigerated in biohazard bags at $2^{\circ} \mathrm{C}$ to $3{ }^{\circ} \mathrm{C}$, and tested within 1 week of collection.

Before testing, all gloves were inspected visually by one author $(\mathrm{EH})$ for macroperforations and no cleaning of potentially biohazardous material was done. Macroperforations were defined as holes $5 \mathrm{~mm}$ or greater, on the assumption that perforations of this size could be seen easily by the surgeon intraoperatively. Any glove that showed macroperforations was not analyzed for other microperforations. Gloves without macroperforations were tested according to the American Society for Testing and Materials standard (ASTM D5151-06) for detection of holes in surgical gloves [3]. Gloves were secured and suspended at the wrist to a rigid polyvinyl chloride pipe and filled with $1 \mathrm{~L}$ water. For 2 minutes, they were observed for breach of integrity. Observance of water on the outer surface of the suspended glove was considered a failed test and these specimens were categorized as having microperforations.

Analyses were performed to determine the percentages of macro- and microperforations, common instruments in 
which the gloves were caught, time of wear, and location of perforation.

\section{Results}

The 33 gloves obtained came from 33 procedures. Seventeen of the 33 (52\%) gloves had perforations; seven (21\%) had visual macroperforations; and $10(30 \%)$ had microperforations that failed the ASTM standard but had not been visually apparent (Fig. 1). No microperforations (perforations $<5 \mathrm{~mm}$ ) were identified by visual inspection before testing (Fig. 2). In total, 10 of 17 of the perforations were not visible.

The most common types of equipment in which the 33 gloves were caught were drills (11), K-wires (eight), and reamers (nine) while the remaining gloves were caught by various other instruments (five). Eight of the 17 perforations were caused by drills, three by reamers, three by K-wires, and three by various other instruments. The most common location reported on gloves caught was the index finger. There were not enough data to show whether any specific glove type had superior perforation resistance. However, all types of gloves submitted (latex, latex-free, orthopaedic) had at least one specimen with microperforations.

Average time worn for all gloves before entanglement was 58 minutes. Gloves without perforations were worn for $51 \pm 41$ minutes. Gloves with macroperforations were worn for $63 \pm 46$ minutes and gloves with microperforations were worn for $68 \pm 42$ minutes. No difference was apparent between time of wear for gloves without perforations and those with macro- or microperforations $(p=0.612)$ (Fig. 3).

\section{Discussion}

Glove integrity is the paramount safety barrier protecting the patient and the surgeon from body fluid exposure or contamination. Similar to understanding side effects and negative consequences of different medicines he or she prescribes, the surgeon must understand the gloves he or she wears when performing a surgery. We aimed to define a mechanism of glove damage to inform surgeons about a previously unreported mechanism of breach of this protective barrier. Our study showed that gloves caught in rotatory power equipment have a high risk of perforation (52\%), with $30 \%$ having microperforations that are not reliably detected with visual inspection. Drills, K-wires, and reamers were the most common instruments associated with glove perforations. Time of wear did not appear to affect the percentage of perforations.

Our study has several limitations. First, gloves were collected on a volunteer basis, therefore we are able to

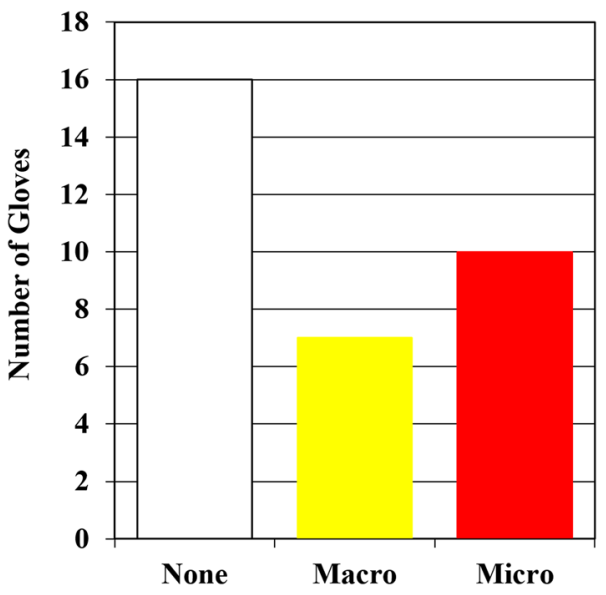

Fig. 1 Gloves tested were categorized by absence (None) or occurrence of macroperforations (Macro) or microperforations (Micro)

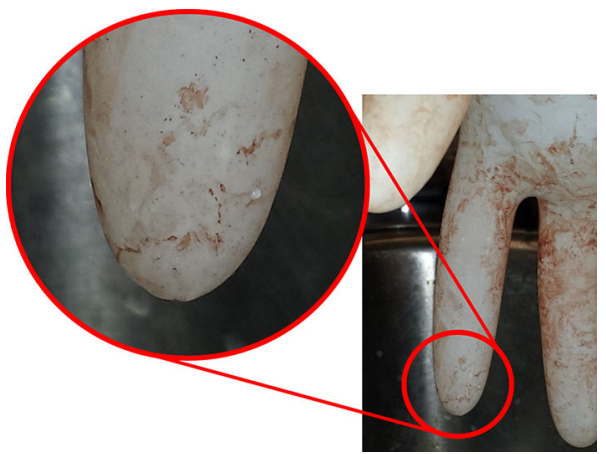

Fig. 2 A microperforation detected as a water droplet during ASTM standard testing is shown.

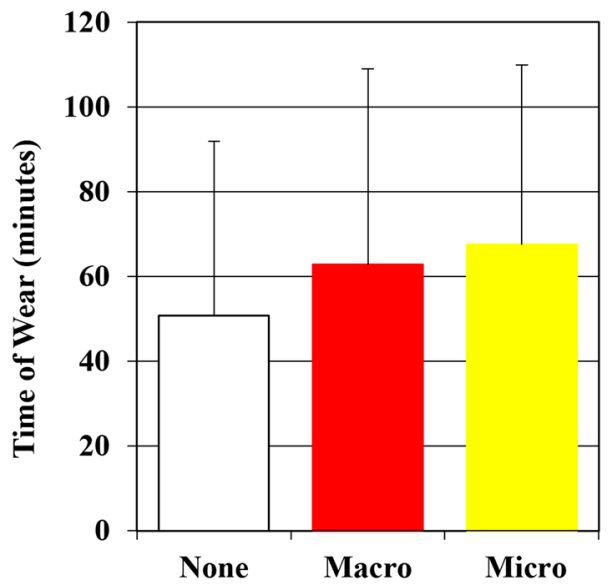

Fig. 3 Time of wear before catching the glove is categorized by absence (None) or occurrence of macroperforations (Macro) or microperforations (Micro).

report only perforation percentage on the gloves that were submitted. The number of gloves collected during the study was fewer than anticipated and we surmise that there were 
likely additional occurrences during the study period. Nonetheless, the data reinforce the idea that surgeons likely do not recognize when loss of glove integrity has occurred by this mechanism. We have no reliable means to estimate incidence of perforation, as the total number of cases in which rotatory tools were used during the study period was not tallied. In addition, the gloves were not cleaned of biologic material before testing, which might have resulted in a lower observance of microperforations, if, for example, coagulated blood was present and formed a seal over a microperforation that remained watertight during testing. Although cleaning the gloves might negate this potential confounder, it conversely could result in additional trauma to the gloves and skew the results. On the topic of microorganisms, our exclusion criteria did not permit analysis of gloves known to have been exposed to transmissible diseases; however, only two gloves were discarded because of this exclusion, therefore it is unlikely to have influenced our findings to any large degree.

We did not have enough data to differentiate perforation risk for different glove types. Different material properties may make a glove more or less prone to shear stress. Hypothetically, latex-free gloves do not have the same hydration effects as latex gloves. However, these gloves do not have the same stringent FDA standards as latex gloves, making direct comparison difficult [19]. With more specimens, more detailed analysis of risk by glove type and other factors could be possible. Double gloving is an effective way to protect patients and practitioners and is common practice in our institution; however, double gloving, while common, is not universally practiced [7, 13, 18]. Mischke et al. [13] found inner-glove puncture risk could be reduced by $71 \%$ with double gloving. We did not evaluate the damage to an inner glove if double gloving was used, as only outer gloves were submitted for testing. In addition, our study primarily was designed to assess glove integrity after exposure to shear from entanglement in a rotatory device, which is a different mechanism than puncture. We are unable to comment on the effects of shear at the interface between glove layers or on the integrity of the inner glove and the potential risk it may pose at this time; likewise we did not have a sufficiently large or diverse sample to compare different types of rotatory instruments, but given the small expense associated with changing gloves, we believe the findings of our study are robust enough to recommend changing gloves after entanglement with any rotatory instrument.

We defined macroperforation as a visually apparent defect of at least $5 \mathrm{~mm}$. While this cutoff was defined arbitrarily, no gloves showing a microperforation had a visually identifiable defect. In addition, our study protocol likely resulted in more meticulous inspection before testing than what is common practice intraoperatively. The percentage of microperforations suggests that visual inspection is inadequate to assess glove integrity and any glove that becomes entangled in a rotatory device should be changed regardless of its appearance. An in vitro control group was considered; however, given the changes in glove properties with time with wear and presence of biologic material, it was not deemed appropriate. An in vivo control also was considered; however, given the known percentages of glove perforations reported in prior studies [2, 4-6] a control group was deemed unnecessary.

The anonymous nature of glove submission allowed the surgeons to submit gloves without risking judgment regarding their surgical ability or care. As such, anonymous submission precluded analysis of risk relative to procedure or specialty. We cannot comment whether our sample size was representative of the entire field of orthopaedics or simply a sample of a few practitioners interested in submitting gloves for the study. The underglove was not studied here, and while one may assume that specialties that use rotatory equipment more than others may be at greater risk, the risk may be surgeon (technique) dependent. This initial study was aimed at defining a glove injury pattern and its associations to raise the question: should the glove be changed? We do not intend to draw more conclusions beyond the answer to that question without a larger study and sample size. This study may generate additional interest in this topic and future study could capture a more representative sample of all the gloves caught on a rotatory device. A larger sample size would allow for multivariable analysis of factors such as the effect of glove type or perforation percentage.

Federal regulations monitor surgical gloves as a medical device. The ASTM one-liter water test (ASTM -D515106) [3] is the gold standard metric for testing medical gloves; therefore, it was the sole test performed on the submitted gloves. Manufacturers are required to randomly test gloves as part of quality assurance during production. The threshold for failure is $2.5 \%$ to meet testing guidelines. Since such testing is random and $2.5 \%$ failure is the accepted standard, it is possible that some of the gloves we tested were defective at the time of manufacture rather than perforated owing to entanglement. While the data from this study suggest that microperforations are present $31 \%$ of the time after entanglement in a rotatory tool, a more-sensitive testing method such as electroconductivity could result in even more concerning findings [17].

The study gloves were submitted at an average wear time of 58 minutes and had an overall perforation risk of $51.5 \%$. Given the average wear time on our glove specimens, one could argue that the risk of damage to gloves seen in this study was attributable merely to chance. Partecke et al. [16] reported that glove perforations increase with the duration of wear owing to mechanical stresses, not 
necessarily attributable to material fatigue. In their study, perforation was observed in $15.4 \%$ of gloves during the first 90 minutes of orthopaedic procedures and this increased to $18.1 \%$ if the glove was worn between 91 to 150 minutes, and to $23.7 \%$ if the glove was worn longer than 150 minutes [16]. Limited data are available regarding risk of compromise of glove integrity in specific orthopaedic procedures $[1,2,4,6,8,9,20]$. At least one glove was found to be perforated in $39 \%$ of TKAs, with a perforation incidence of $4.29 \%$ of the gloves worn in these procedures [8]. The mechanism of contamination was not reported in that study. Likewise in orthopaedic trauma, Eckersley and Williamson [6] found that a single glove may be perforated more than $50 \%$ of the time during the course of internal fixation of a fracture and this rate was reduced to $17 \%$ with double gloving. Our study suggests that entanglement in rotatory tools is a mechanism in which perforations have a greater likelihood of occurrence. Few studies have evaluated the specific mechanisms in which glove perforations occur, except needle sticks [4-6, 9]. Chan et al. [5] showed that perforations are caused by instrumentation in $45 \%$ of all glove perforations, versus only $20 \%$ by bone debris, $15 \%$ by surgical knots, and only $5 \%$ are attributable to scalpel blades or pins each. Martinez et al. [11] showed in an in vitro model that knot tying with high tensile strength suture causes a relatively low risk (3.4\%) of glove perforations.

While limitations of our study make it clear that we have not proven that a hole in the glove results in communication between the environment and the surgeons' hands, and certainly not that communication with the hands will result in infection, the cost of replacing gloves after being caught in a rotatory instrument seems small in comparison to the risk, and we found a higher risk of perforations than one might expect. Because of this, we believe the results of the current study suggest that any glove that becomes entangled in a rotatory device should be changed immediately, regardless of the surgeon's visual assessment of glove integrity. Our visual inspection was more stringent than what typically is done intraoperatively, which suggests that surgeons underestimate glove perforation. More than $1 / 2$ of the gloves in this study that became entangled in a rotatory device sustained sufficient damage to cause a perforation and 10 of 17 such occurrences were not visible. Future studies are needed to compare different glove types and the risk to inner gloves when rotatory entanglement occurs.

\section{References}

1. Al-Habdan I, Sadat-Ali M. Glove perforation in pediatric orthopedic practice. J Pediatr Orthop. 2003;23:791-793.
2. Al-Maiyah M, Bajwa A, Mackenney P, Port A, Gregg PJ, Hill D, Finn P. Glove perforation and contamination in primary total hip arthroplasty. J Bone Joint Surg Br. 2005;87:556-559.

3. ASTM International. ASTM D5151-06(2015), Standard Test Method for Detection of Holes in Medical Gloves, ASTM International, West Conshohocken, PA, 2015. DOI: 10.1520/ D5151-06R15. Available at: http://www.astm.org/Standards/ D5151.htm. Accessed March 1, 2016.

4. Beldame J, Lagrave B, Lievain L, Lefebvre B, Frebourg N, Dujardin F. Surgical glove bacterial contamination and perforation during total hip arthroplasty implantation: when gloves should be changed. Orthop Traumatol Surg Res. 2012;98:432-440.

5. Chan KY, Singh VA, Oun BH, To BH. The rate of glove perforations in orthopaedic procedures: single versus double gloving: a prospective study. Med J Malaysia. 2006;61 (suppl B):3-7.

6. Eckersley JR, Williamson DM. Glove punctures in an orthopaedic trauma unit. Injury. 1990;21:177-178.

7. Haines T, Stringer B, Herring J, Thoma A, Harris KA. Surgeons' and residents' double-gloving practices at 2 teaching hospitals in Ontario. Can J Surg. 2011;54:95-100.

8. Han CD, Kim J, Moon SH, Lee BH, Kwon HM, Park KK. A randomized prospective study of glove perforation in orthopaedic surgery: is a thick glove more effective? $J$ Arthroplasty. 2013;28: 1878-1881.

9. Kaplan KM, Gruson KI, Gorczynksi CT, Strauss EJ, Kummer FJ, Rokito AS. Glove tears during arthroscopic shoulder surgery using solid-core suture. Arthroscopy. 2007;23:51-56.

10. Lemaire R, Masson JB. Risk of transmission of blood-borne viral infection in orthopaedic and trauma surgery. J Bone Joint Surg Br. 2000;82:313-323.

11. Martinez A, Han Y, Sardar ZM, Beckman L, Steffen T, Miller BS, Martineau PA. Risk of glove perforation with arthroscopic knot tying using different surgical gloves and high-tensile strength sutures. Arthroscopy. 2013;29:1552-1558.

12. McCue SF, Berg EW, Saunders EA. Efficacy of double-gloving as a barrier to microbial contamination during total joint arthroplasty. J Bone Joint Surg Am. 1981;63:811-813.

13. Mischke C, Verbeek JH, Saarto A, Lavoie MC, Pahwa M, Ijaz S. Gloves, extra gloves or special types of gloves for preventing percutaneous exposure injuries in healthcare personnel. Cochrane Database Syst Rev. 2014;3:CD009573.

14. Misteli H, Weber WP, Reck S, Rosenthal R, Zwahlen M, Fueglistaler P, Bolli MK, Oertli D, Widmer AF, Marti WR. Surgical glove perforation and the risk of surgical site infection. Arch Surg. 2009;144:553-558; discussion 558.

15. Myers DJ, Epling C, Dement J, Hunt D. Risk of sharp devicerelated blood and body fluid exposure in operating rooms. Infect Control Hosp Epidemiol. 2008;29:1139-1148.

16. Partecke LI, Goerdt AM, Langner I, Jaeger B, Assadian O, Heidecke CD, Kramer A, Huebner NO. Incidence of microperforation for surgical gloves depends on duration of wear. Infect Control Hosp Epidemiol. 2009;30:409-414.

17. Sohn RL, Murray MT, Franko A, Hwang PK, Dulchavsky SA, Grimm MJ. Detection of surgical glove integrity. Am Surg. 2000;66:302-306.

18. Tanner J, Parkinson H. Double gloving to reduce surgical crossinfection. Cochrane Database Syst Rev. 2006;3:CD003087.

19. US Food and Drug Administration. CFR - Code of Federal Regulations Title 21. Patient examination gloves and surgeons' gloves; sample plans and test method for leakage defects; adulteration. Available at: http://www.accessdata.fda.gov/scripts/ cdrh/cfdocs/cfcfr/CFRSearch.cfm?fr=800.20. Accessed March 1, 2016.

20. Yinusa W, Li YH, Chow W, Ho WY, Leong JC. Glove punctures in orthopaedic surgery. Int Orthop. 2004;28:36-39. 